\title{
When Helping Babies Breathe Is Not Enough: Designing a Novel, Mid-Level Neonatal Resuscitation Algorithm for Médecins Sans Frontières Field Teams Working in Low-Resource Hospital Settings
}

\author{
Lisa Umphrey ${ }^{\mathrm{a}} \quad$ Morten Breindahl $^{\mathrm{b}} \quad$ Alexandra Brown $^{\mathrm{c}}$ Ola Didrik Saugstad ${ }^{\mathrm{d}}$ \\ Marta Thio $^{\text {e }}$ Daniele Trevisanuto ${ }^{f}$ Charles Christoph Roehrg Mats Blennow ${ }^{b}$

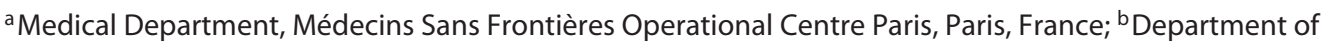 \\ Neonatology, Karolinska Institutet and University Hospital, Stockholm, Sweden; ${ }^{\circ}$ Communications Department, \\ Médecins Sans Frontières, Sydney, NSW, Australia; ${ }^{d}$ Department of Pediatric Research, Oslo University Hospital \\ and University of Oslo, Oslo, Norway; ${ }^{~ N e w b o r n ~ R e s e a r c h ~ C e n t r e, ~ T h e ~ R o y a l ~ W o m e n ' s ~ H o s p i t a l ~ a n d ~ U n i v e r s i t y ~ o f ~}$ \\ Melbourne, Melbourne, VIC, Australia; ${ }^{f}$ Department of Woman's and Child's Health, University of Padova, Padova, \\ Italy; ${ }^{9}$ Department of Paediatrics, Newborn Services, John Radcliffe Hospital, Oxford University Hospitals, NHS \\ Foundation Trust, University of Oxford, Oxford, UK
}

\section{Keywords}

Algorithm · Bag-and-mask ventilation · Flowchart .

Guideline · Low-resource setting · Newborn · Resuscitation

\begin{abstract}
Background: Neonatal resuscitation (NR) combines a set of life-saving interventions in order to stabilize compromised newborns at birth or when critically ill. Médecins Sans Frontières/Doctors Without Borders (MSF), as an international medical-humanitarian organization working particularly in low-resource settings (LRS), assisted over 250,000 births in obstetric and newborn care aid projects in 2016 and provides thousands of newborn resuscitations annually. The Helping Babies Breathe (HBB) program has been used as formal guidance for basic resuscitation since 2012. However, in some MSF projects with the capacity to provide more advanced NR interventions but a lack of adapted guidance, staff have felt prompted to create their own advanced algo-
\end{abstract}

\begin{tabular}{ll}
\hline KARGER & $\begin{array}{l}\text { (c) } 2018 \text { The Author(s) } \\
\text { Published by S. Karger AG, Basel }\end{array}$ \\
E-Mail karger@karger.com & $\begin{array}{l}\text { This article is licensed under the Creative Commons Attribution- } \\
\text { NonCommercial-NoDerivatives 4.0 International License (CC BY- } \\
\text { NC-ND) (http://www.karger.com/Services/OpenAccessLicense). } \\
\text { Usage and distribution for commercial purposes as well as any dis- } \\
\text { tribution of modified material requires written permission. }\end{array}$
\end{tabular}

rithms, which runs counter to the organization's aim for standardized protocols in all aspects of its care. Objectives: The aim is to close a significant gap in neonatal care provision in LRS by establishing consensus on a protocol that would guide MSF field teams in their practice of more advanced NR. Methods: An independent committee of international experts was formed and met regularly from June 2016 to agree on the content and design of a new NR algorithm. Results: Consensus was reached on a novel, mid-level NR algorithm in April 2017. The algorithm was accepted for use by MSF Operational Center Paris. Conclusion: This paper contributes to the literature on decision-making in the development of cognitive aids. The authors also highlight how critical gaps in healthcare delivery in LRS can be addressed, even when there is limited evidence to guide the process.

(C) 2018 The Author(s)

Published by S. Karger AG, Basel

Charles Christoph Roehr and Mats Blennow share senior authorship.
Lisa Umphrey

Médecins Sans Frontières Australia PO Box 847

Broadway, NSW 2007 (Australia)

E-Mail lisa.umphrey@ sydney.msf.org 


\section{Background}

Neonatal resuscitation (NR) combines a set of life-saving interventions in order to stabilize compromised newborns at birth or when critically ill. As an emergency response, NR places considerable pressure on the providers' knowledge and experience in a short space of time [1]. Therefore, NR is classified as a high-acuity, low-occurrence skill: one that requires ongoing practice and learning to maintain the skills acquired in its initial teaching $[2,3]$. Current international guidelines and recommendations advise on effective interventions and the sequence in which they should ideally be provided $[4,5]$. NR training can improve teamwork [6] and significantly reduce neonatal mortality [7]. Therefore, programs for teaching $\mathrm{NR}$, based on the best available evidence as reviewed in the abovementioned guidance, are now being taught worldwide. Examples of such programs catering to highresource settings (HRS) include Newborn Life Support by the European Resuscitation Council (ERC) and the Neonatal Resuscitation Program (NRP) from the American Academy of Pediatrics (AAP) [8-10]. Helping Babies Breathe (HBB), also from the AAP, has been shown to be an effective and well-accepted NR program in low-resource settings (LRS) $[3,11,12]$.

Médecins Sans Frontières/Doctors Without Borders (MSF), an international medical-humanitarian organization working particularly in LRS, assisted over 250,000 births in obstetric and newborn care aid projects in 2016 [13]. The HBB protocol has been well adopted as formal guidance for basic resuscitation in MSF projects since 2012. However, HBB is especially targeted at health facilities with virtually no capacity to provide advanced NR interventions [11], which is not the case for most MSF projects. In MSF hospitals, although there is usually no physician or pediatric specialist present in the delivery room specifically for the neonate, there is always a midwife with the ability to focus on the baby and call for additional help. In the newborn unit it is routine to have a mid-level healthcare provider or general physician, and often there is a pediatrician on at least a part-time basis. Teams in both of these clinical areas have routine access to basic resuscitation equipment, a pulse oximeter and oxygen, but not to more technical support such as highflow nasal cannula, continuous positive airway pressure, or mechanical ventilation.

The availability of trained staff and of basic resuscitation equipment allows for NR interventions beyond the main focus of the HBB program (face mask ventilation in room air), but not as extensive as recommended for HRS

Designing a Mid-Level Neonatal

Resuscitation Algorithm for MSF
[4]. Lacking alternative guidance for a mid-level setting, MSF field staff have often felt prompted to create their own advanced algorithms to address their teams' higher capacity. Yet such practice runs counter to the organization's aim for standardized, although at the same time context-adapted, protocols in all aspects of its care in order to achieve consistency in training and clinical practice in the field.

It was therefore decided to attempt to establish consensus on a protocol that would guide MSF field teams in their practice of more advanced NR, adapted for LRS. To achieve this, an independent committee of international experts was formed and met regularly from June 2016, deliberating throughout on how best to meet MSF's needs. This report describes the iterative process that led to agreement on the content and design of the novel algorithm in April 2017, and successful acceptance for use in MSF Operational Center Paris (MSF-OCP) hospital projects. In describing this collaborative process and its positive outcome, the authors hope to shine a light on how critical gaps in healthcare delivery in resource-poor settings can be addressed, even when little formal medical literature is available to guide the process.

\section{Forming the Working Group and Setting Its Goals}

Although MSF's medical projects are often located in LRS, the level of resources that can be allocated to programs means that there is the capacity to provide a more advanced level of care. Under the initiative of authors L.U. and M.Bl., an international group of experts agreed to collaborate pro bono to develop a refined resuscitation algorithm for newborns in the care of MSF. Members of the group brought a range of complementary experience, including MSF and other LRS experience, protocol development in LRS and HRS, critical appraisal of the current medical literature, research on different components of $\mathrm{NR}$, and expertise in newborn intensive care units in their home countries.

The group first convened in June 2016 via a videoconference call. Subsequent meetings were also conducted online using online video conferencing (Skype, Luxembourg City, Luxembourg) and GoToMeeting (Santa Barbara, CA, USA), connecting the group's members in Australia, Italy, the UK, Norway, and Sweden.

L.U. acted as the record-keeper and algorithm designer. Minutes were documented for every meeting, and action points assigned. Discussion was conducted by email or phone in between meetings. 
The group coalesced behind 3 key objectives: to identify gaps in current low- and high-capacity NR algorithms, to recommend how to address them, and to adapt an algorithm as the solution, based on expert consensus. This "adapted moderate-capacity NR algorithm" would be novel in the level of intensity of the provided treatment, yet suitable for resuscitation in areas of limited resources - calibrated above HBB but below HRS algorithms derived from the recommendations of the International Liaison Committee on Resuscitation (ILCOR) [4]. Specific goals were then plotted under these objectives, as shown in Table 1.

\section{The Deliberative Process}

Several meetings were held with extensive email discussion in between, before the first version of the algorithm was drafted. Debate largely focused on the benefits and challenges of the HBB protocol as experienced in MSF settings.

HBB was first developed in 2010 and marked a breakthrough in the development of programs for newborn care adapted to LRS [14]. In 2016, a second edition was released to bring it in line with the 2015 ILCOR Consensus on Science with Treatment Recommendations and the 2012 WHO Guidelines on Basic Newborn Resuscitation $[4,11,15]$. There were 3 main practical improvements in the revised HBB package - new limitations to suctioning, prioritization of the decision to ventilate prior to cord-cutting, and skills consolidation after implementation - but it remained, understandably, focused on the simplest and highest impact aspects of NR $[9,16]$ and not on advanced steps past effective ventilation.

Initial discussions also focused on the generic ILCOR algorithm for NR, a template for the design of contextspecific resuscitation algorithms that is meant to be adapted to the patient population and resources $[2,4]$. A recent modified DELPHI review of the ILCOR and 4 adapted HRS algorithms found the former slightly more effective than the adaptations by ILCOR members [2]. The group agreed that the template was simple and clear; it was therefore felt to be a good base for the group's new algorithm. However, with its discussion of intubation, continuous positive airway pressure, and electrocardiography monitoring, the ILCOR algorithm is inherently oriented towards HRS [17].

The authors thus began the first draft, seeking, on the one hand, to be as much aligned with existing evidencebased algorithms and recommendations as possible.
Table 1. Objectives and goals of the working group for a mid-level NR algorithm for LRS

Working group: adapted moderate-capacity neonatal resuscitation algorithm. Objectives and Goals. June 2016.

Objectives

Identify gaps in current low- (HBB) and high- (NRP) capacity neonatal resuscitation algorithms

Compile expert recommendations on how to address these gaps in LRS with moderate resources and human resource capacity Create an adapted neonatal resuscitation algorithm for these particular settings based on expert consensus

Specific goals

Review existing "high-capacity" algorithms

European Resuscitation Council (ERC)

NRP (through the American Academy of Pediatrics, AAP)

Australian Resuscitation Council (ARC)

Review existing "low-capacity" algorithms

HBB (AAP)

Review examples of "moderate-capacity" algorithms used in LRS such as MSF fields

Discuss results of literature review for any adapted-up (HBB-plus

type tools) or adapted-down (NRP-minus type tools)

resuscitation techniques in moderate-capacity settings

Compile a proposed moderate-capacity algorithm

Test the algorithm's feasibility in MSF fields in partnership with Ministries of Health

Validate the algorithm on behalf of MSF and involved partners Incorporate the algorithm into MSF guidelines and other clinical venues as per the group's wishes

HBB, Helping Babies Breathe; NRP, Neonatal Resuscitation Program; LRS, low-resource settings; MSF, Médecins Sans Frontières.

Conversely, in appreciation that much of the current evidence for delivery room resuscitation was derived from research in HRS, it was necessary to leave out points specific to HRS, and, in recognition of the role of human and contextual factors, to incorporate design elements and content to address the specific challenges in MSF projects [2]. The foundation was the ILCOR algorithm with the accepted airway, breathing, and circulation - ABC - approach [16]. Discussions then concentrated on several key elements felt to be important for a mid-level algorithm, such as the use of inflation breaths $[16,18]$, when to check the heart rate, when or if to suction for meconium, how or when to evaluate and treat hypoxemia, and the provision of chest compressions. These points were incorporated in the first draft, shown in Figure 1.

Every step was first designated as either an Action (black box), a Question (red stop sign), or a Final Action (blue box). All paths would reach a definitive end-point, 


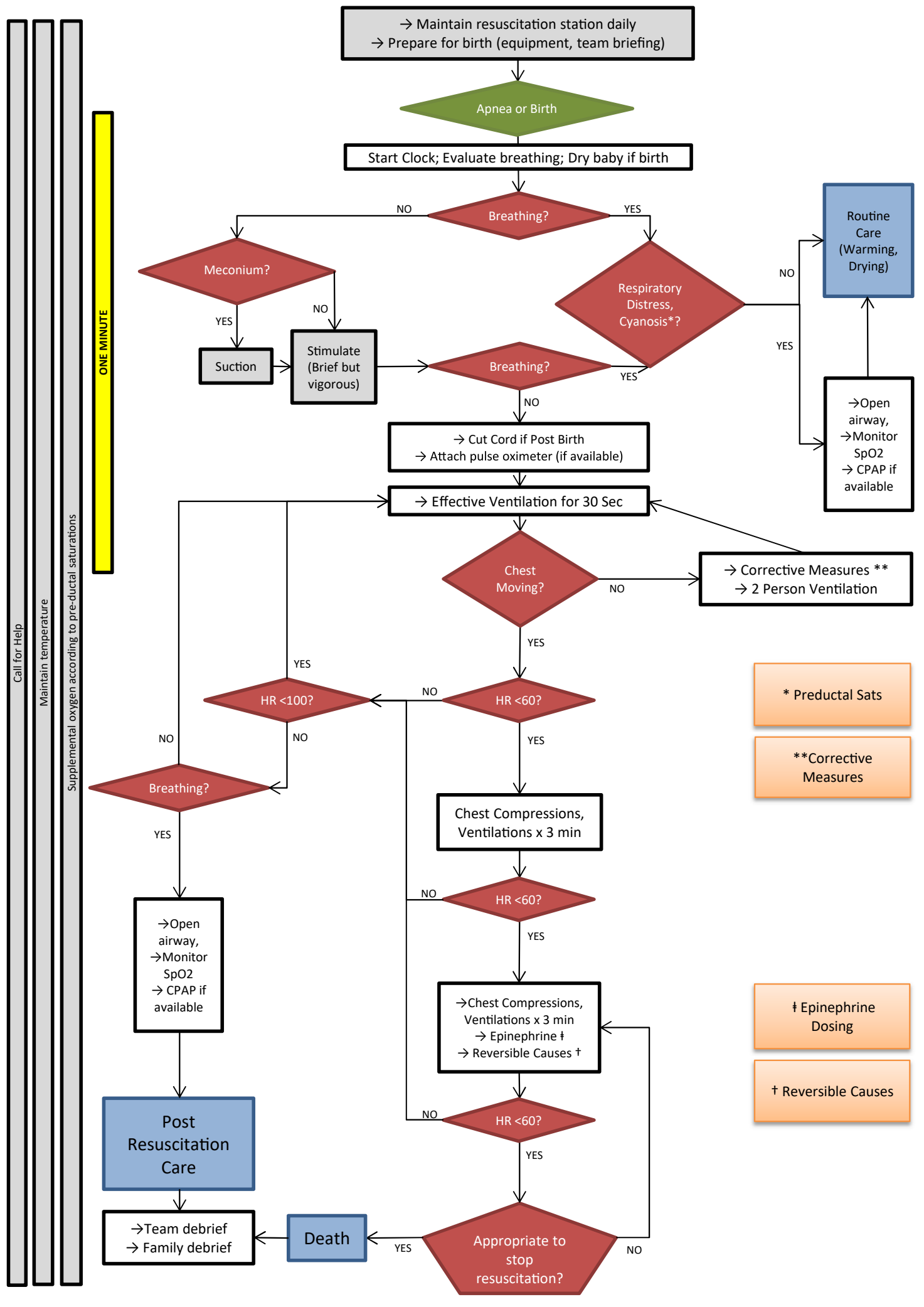

Fig. 1. Progression of the NR algorithm throughout the consensus process: draft 1 of a mid-level algorithm for NR in LRS. 


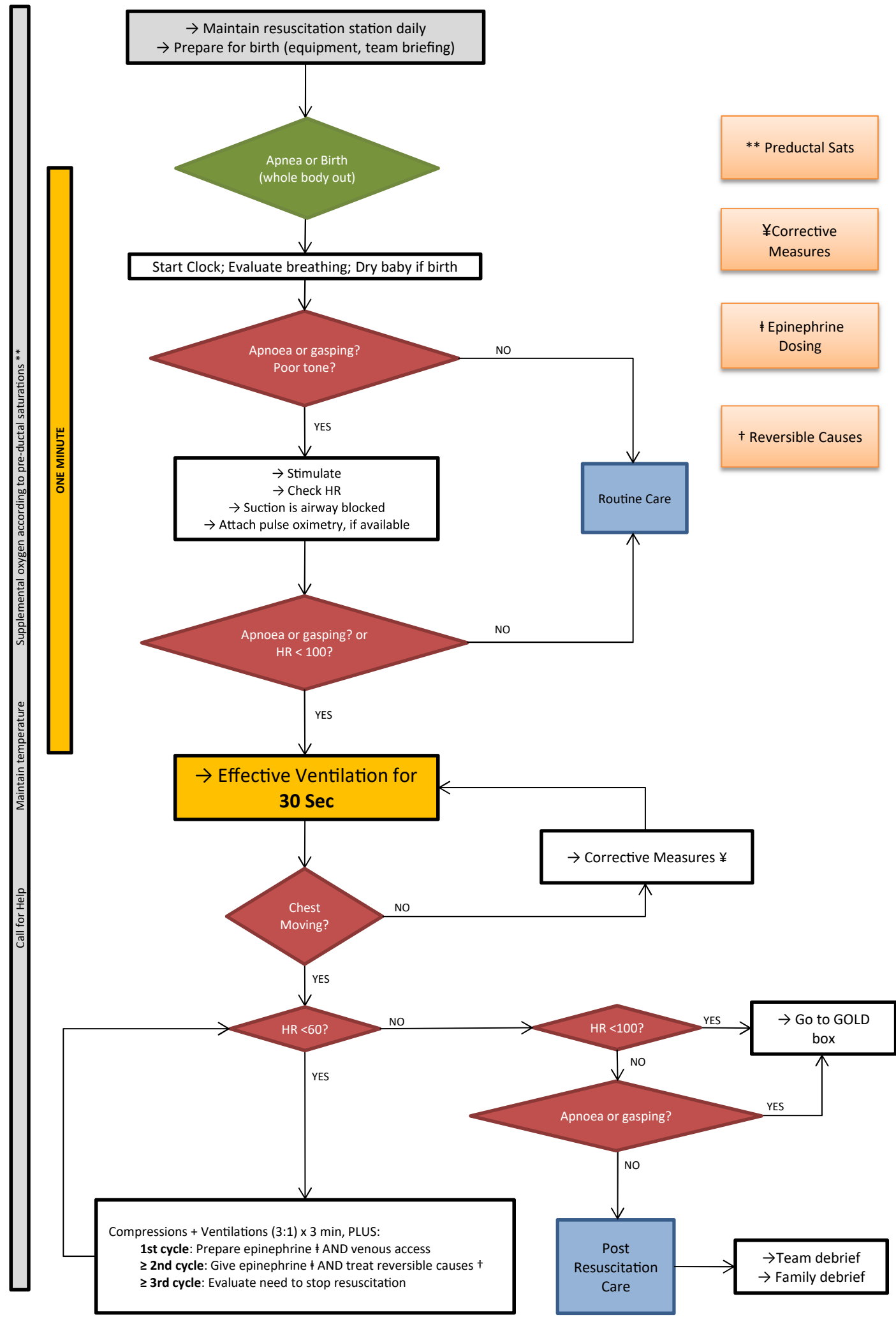

Fig. 2. Progression of the NR algorithm throughout the consensus process: draft 2 of a mid-level algorithm for NR in LRS. 
to avoid creative interpretation or getting lost if users were not accustomed to algorithm use. Language was kept simple where appropriate; for example, "breathing, not breathing/crying" replaced "apnea/gasping." Where possible, pathways were cyclical, to include ongoing rechecks of breathing, heart rate, and chest rise. More specifically, a detailed cycle was incorporated in the flowchart to remind users to focus on effective ventilation before moving forward; this is a well-documented and serious problem in the provision of NR [3] which circumvents the efficacy of any subsequent actions. Ranges were avoided overall for clarity; for example, chest compressions were limited to $3 \mathrm{~min}$, not 3-5 min, to be clear and to better match with the chronology of epinephrine doses. Lastly, in accord with HBB recommendations and the current medical literature, an outcome for stopping resuscitation and death was included and linked to a debrief of both the team and family [19].

This first draft was informally checked for effectiveness against the cognitive aids in medicine assessment tool (CMAT) [2] and Marshall's 4-point [1] criteria by L.U. before presentation to the group. This initial draft elicited intense but very productive feedback focusing on the volume of content, the necessity for certain elements, and the best manner to present the key points. The second draft thus incorporated significant changes, notably streamlined pathways (Fig. 2), but much of the thinking and flow that was established at this stage remained relevant throughout subsequent versions.

After the second draft, most decisions regarding the content of the algorithm were couched within the concern to arrive at the ventilation step within the first minute of life [8]. Consequently, every successive point was considered in this decisional context. Iterations from the third draft onward were driven by avoiding or prudently placing tasks that took disproportionately long amounts of time in MSF field settings (evaluating tone; drying followed by stimulation, instead of at the same time; evaluating heart rate) to force users to arrive as quickly as possible at ventilation.

As the consensus further developed the principal content decisions centered on 6 factors: thermal losses; delayed cord clamping; suctioning of meconium; establishing effective ventilation; supplemental oxygen; and stopping resuscitation. The rationale for each is outlined in Table 2; the topics are organized by chronologic order in the algorithm, not by importance or degree of debate undertaken for each.

Over a 10-month period from July 2016, the group generated more than 10 drafts of the algorithm before reaching its final consensus. By April 2017, the singlepage algorithm was ready to be reviewed by pediatric advisors and MSF field teams (Fig. 3).

\section{Incorporating Field Input}

To fulfill the feasibility testing goal originally set by the committee, albeit in a preliminary fashion, informal usability testing was conducted amongst 3 MSF pediatric teams, 1 French-speaking in Ivory Coast and 2 Englishspeaking, in Pakistan and South Sudan. The 2-week period consisted of group discussions on the algorithm's content and use during several resuscitations (3-6 per site) by a clinical team under the supervision of an MSF pediatrician. This preliminary feedback overturned some of the committee's earlier decisions on logic and format, and called for more clarity. There were 4 main points, as outlined below.

Yes/no orientation: "no" needed to replace "yes" as the impetus to progress through the algorithm, to address the idea that moving forward in the algorithm is "negative" and equates with the baby not doing well.

Cycles of advanced resuscitation: the expert group agreed from the beginning on the need to include compressions/adrenaline. The focus was therefore how best to include this information, and the result was the box included in the draft submitted to the field projects (Fig. 3). Field teams, however, were still confused, which led to clarification of what should happen in each cycle, and reinstatement of words to further explain the steps more clearly.

Routine care and postresuscitation care text boxes: originally considered important to include, they were uniformly judged unnecessary by the field teams (because the information is included elsewhere, in MSF's neonatal guidelines).

Birth box: in earlier iterations, "caveats" in each box accounted for when the algorithm was effectuated during a birth; field teams felt this to be confusing and made the logical suggestion to have a separate box, concurrent and parallel, to address all birth considerations at once.

\section{Results}

MSF's priority was to have a practical tool available for its neonatal projects, and eventually included in the MSF intersectional neonatal guidelines (expected publication in 2018). This was to be independent of training 
Table 2. Key content decisions for the novel algorithm and their rationale

\begin{tabular}{|c|c|c|c|}
\hline Issue & MSF relevance & Outcome & Rationale \\
\hline Thermal losses & $\begin{array}{l}\text { Radiant warmers are routinely provided in } \\
\text { delivery rooms and newborn units in MSF } \\
\text { hospitals } \\
\text { Hypothermia is an important issue in MSF } \\
\text { projects }\end{array}$ & $\begin{array}{l}\text { A vertical bar saying "prevent heat loss" } \\
\text { spans the whole algorithm }\end{array}$ & $\begin{array}{l}\text { Preventing thermal losses in the neonate is an } \\
\text { important transversal consideration }[4,16]\end{array}$ \\
\hline $\begin{array}{l}\text { Delayed cord } \\
\text { clamping }\end{array}$ & $\begin{array}{l}\text { Reduction in anemia has significant } \\
\text { implications for patients at high risk in } \\
\text { LRS } \\
\text { MSF teams do not always consider delayed } \\
\text { cord clamping while resuscitating a } \\
\text { neonate } \\
\text { NR occurs anytime during the neonatal } \\
\text { period in MSF projects, not just at birth }\end{array}$ & $\begin{array}{l}\text { Initially included as "consider delayed cord } \\
\text { clamping" in the first action box } \\
\text { After receiving field feedback on the final } \\
\text { draft, all birth-specific steps were } \\
\text { incorporated to a parallel box at the } \\
\text { beginning, limiting emphasis of its content } \\
\text { to mention its necessity: (1) concurrently } \\
\text { to other steps and (2) within the 1-min } \\
\text { limit }\end{array}$ & $\begin{array}{l}\text { Current evidence advises delayed cord } \\
\text { clamping after birth, as it is linked to positive } \\
\text { consequences in the short and long term [ } 4 \text {, } \\
20-25] \text {; however, the frequent lack of expertise } \\
\text { in MSF field teams necessitated balancing the } \\
\text { advice (which relies on accurate assessment of } \\
\text { the baby's level of "depression" and need for } \\
\text { resuscitation) with the need to provide } \\
\text { effective ventilation within } 1 \text { min }\end{array}$ \\
\hline $\begin{array}{l}\text { Suctioning of } \\
\text { the mouth and } \\
\text { oropharynx }\end{array}$ & $\begin{array}{l}\text { Over-suctioning is a common issue in } \\
\text { MSF settings, which results in a delayed } \\
\text { start to ventilation } \\
\text { Intubation equipment is not routine in } \\
\text { MSF projects, eliminating tracheal } \\
\text { suctioning of meconium as a concern }\end{array}$ & $\begin{array}{l}\text { Instruction to NOT suction unless there is } \\
\text { thick meconium AND no breathing or } \\
\text { crying }\end{array}$ & $\begin{array}{l}\text { The consensus committee discussed how to } \\
\text { balance instructions on suctioning when } \\
\text { necessary, versus not wasting too much time, } \\
\text { versus recommendations to limit the } \\
\text { suctioning of meconium }[9,16] \text {; as per the } 2 \text { nd } \\
\text { edition of HBB, suctioning of meconium early } \\
\text { in resuscitation is no longer indicated as } \\
\text { evidence shows that it delays the start of } \\
\text { ventilation; as a result, all suctioning was } \\
\text { included in the algorithm but with the caveat } \\
\text { to provide a "negative" instruction regarding } \\
\text { when not to suction }\end{array}$ \\
\hline
\end{tabular}

Establishing effective ventilation
NR often progresses in accordance with an algorithm despite effective ventilation having never been assured Many MSF field teams are not experienced in NR technical skills and thus do not provide effective ventilation MSF teams are not always able to accurately assess heart rate, which therefore cannot be used to accurately guide ventilation

Supplemental Regular access to pulse oximetry and oxygen

Teams often focus on measuring or
starting oxygen at the expense of other
higher-impact resuscitative efforts
Teams care for both pre- and full-term
neonates

\begin{tabular}{ll}
\hline Stop & High activity in MSF projects means large \\
resuscitation & numbers of babies require resuscitation \\
& each year \\
& An expected proportion of neonates do \\
& not respond to resuscitation \\
& Advanced interventions rarely provided to \\
& critically ill neonates \\
& HBB recommendations for the duration of \\
& resuscitation already implemented and \\
& widely accepted in MSF fields
\end{tabular}

The "Effective Ventilation" step was made the most visually apparent Chest rise, not heart rate, as the indicator of whether ventilations are initially effective

A loop based on chest rise instructs providers to "go no further" until ventilation includes chest rise Instructions on the most important corrective measures, including tactics not always addressed within other HRS programs (2-person ventilation, oral airway), were incorporated into the pertinent loop in order of importance Addition of a small caveat limiting this cycle to avoid a permanent, ineffective loop

Oxygen only mentioned after effective ventilation has been addressed Oxygen use linked to pulse oximetry, and via text box, to normal saturations per minute of life

Keeping in mind that ventilation is the most important step in NR $[3,4,9,16,24]$, a feedback cycle was considered by MSF as one of the most important features for the new algorithm

A balance was required between instructing teams to "go no further" until they knew ventilation was successful, and trapping teams in an endless loop of "not doing anything" should ventilation not be working

It was also discussed how to tell teams that ventilation was successful (checking for rises in heart rate, the usual indicator, versus watching for chest rise, an acceptable indicator of proper ventilation) and to provide concrete steps for correcting ineffective ventilation

In HRS, although room air is increasingly preferred to supplemental oxygen during NR $[9,16,22,26]$, pulse oximetry and supplemental oxygen are established interventions, particularly for premature neonates, and routinely included in resuscitation algorithms when proper monitoring of saturations is assured

HBB recommendations included twice for emphasis, once in the algorithm and again in a clarifying text box on the side
Since roll-out, HBB has included specifications regarding when to stop resuscitation There is also much evidence to suggest that even in HRS, resuscitations past $10 \mathrm{~min}$ if there is no HR, and $20 \mathrm{~min}$ if there is no recovery of spontaneous circulation, do not contribute to better outcomes $[4,27-31]$ and that clinicians should recognize the inevitability of death $[19,32]$

NR, neonatal resuscitation; HBB, Helping Babies Breathe; LRS, low-resource settings; HRS, high-resource settings; MSF, Médecins Sans Frontières. 


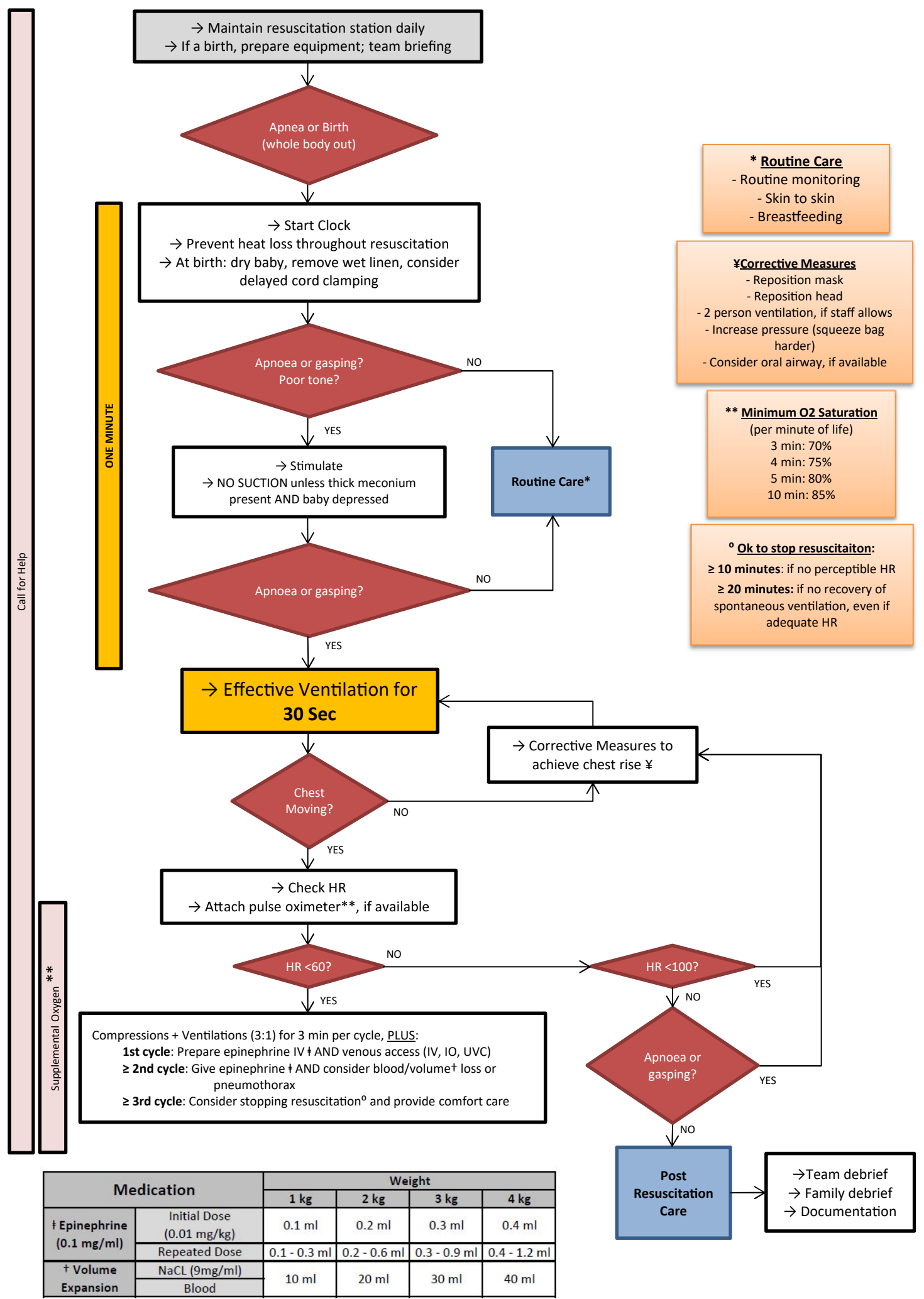

Fig. 3. Progression of the NR algorithm throughout the consensus process: draft 7 of a mid-level algorithm for NR in LRS.

Designing a Mid-Level Neonatal Resuscitation Algorithm for MSF
Neonatology 2018;114:112-123

DOI: $10.1159 / 000486705$ 
supplements or programs or formal field testing, which will be structured around the finalized algorithm. This has been achieved, and the result is a functional, midcapacity protocol for advanced NR in LRS that can be used both at birth and for sick infants in newborn units. This is the most unique feature of the new algorithm (Fig. 4).

As noted to be problematic with other algorithms [2], the new algorithm represents a normal flow of expected events during resuscitations and does not necessarily reflect all contingencies, which can be variable in MSF field settings. Furthermore, it is branched, not linear, which is also thought to be a limitation [1]; however, branching is accepted as appropriate in the case of unstable newborns not assumed to follow a certain clinical course during resuscitation [2].

As opposed to others, the new algorithm includes visual instructions for cyclic evaluation of the efficacy of ventilation, chest compressions, dosing of adrenaline, "negative" words in the algorithm to relay instructions (i.e., to not suction baby unless certain conditions are met), and end-points, which are all crucial to care provision in LRS where advanced supportive care is not possible. These are all noted to be positive elements in an algorithm [2].

The algorithm also supports team co-ordination (calling for help, discussions of 2-person ventilation, team briefing and debriefing), which is considered valuable to enhance the effectiveness of any cognitive aid $[1,6,7]$. At a more fundamental design level, the flowchart has no illustrations to show how to complete the tasks; this helped expedite consensus on the practical content, and readiness for implementation in the field. Nonetheless, given the highly positive acceptance of HBB with its illustrations $[9,3]$, this is something to address in the future.

Colors have been used but within a limited palette as the algorithm will, in many cases, be printed in black and white on A4 paper. Question boxes (strictly speaking, diamonds), however, have remained red, which will allow differentiation even in grayscale.

Based on the expert consensus process, MSF-OCP accepted to use and formally field test the algorithm in its field projects, and the algorithm will be included in MSF's inaugural intersectional neonatal guidelines. As a first step, the algorithm has been reproduced and disseminated as wall charts for MSF projects, in pdf format online within MSF's online portal, and in the aforementioned 2018 neonatal guidelines.

As the algorithm is rolled out in MSF projects, authors will continue to gather both formal and informal feedback regarding the tool's usability and acceptance in the field; we anticipate ongoing monitoring and evaluation to ensure quality assurance recommendations [22]. Improvements and updates to the algorithm are likewise anticipated through internal and external input, including from ministries of health and research boards in MSF project countries. Formal assessment utilizing a CMAT tool or a modified DELPHI review with methodology similar to previous studies [3] will also be valuable to evaluate the algorithm's use overall and its ongoing validity in MSF field settings as feedback is incorporated.

Since mid-2017, a specific neonatal emergency hospital training program has provided details about how to follow the algorithm and implement it in MSF settings; training is a crucial component of any clinical visual aid and necessary to capitalize on potential reductions in mortality [33]. After the tool's initial rollout, further training materials will be created as needed per field feedback. It is also recognized that adaptation to a tablet or smart phone application has the potential to improve the cognitive effect when compared to a traditional visual algorithm [34], and this will be considered as MSF investigates these possibilities in the field.

\section{Conclusion}

This paper showcases an important gap in neonatal care provision in low-resource contexts - the lack of an evidence-based, mid-level NR algorithm where there are sufficient resources and expertise, such as for MSF field workers who are able to provide advanced NR and limited postresuscitation care as a step beyond $\mathrm{HBB}$. This gap, in settings where providing full advanced resuscitation interventions according to ILCOR and ERC recommendations is not feasible, has led to the lack of a standardized, higher level of care for newborns suffering respiratory failure within and outside of the delivery room.

We have described the collaborative expert consensus process undertaken by a group of neonatologists and MSF clinicians to find a solution and, in doing so, contribute to closing the literature gap on decision-making in the development of cognitive aids.

We seek to stimulate further discussion on the role of this algorithm and encourage its use. Lastly, we hope that by documenting the process we have managed to provide some guidance on how to address problems for which there is no or little evidence in the medical literature, or for a unique clinical situation like an MSF project. 


\section{Advanced Neonatal Resuscitation for Low-Resource Settings}

To be used both for sick infants in newborn units and at birth

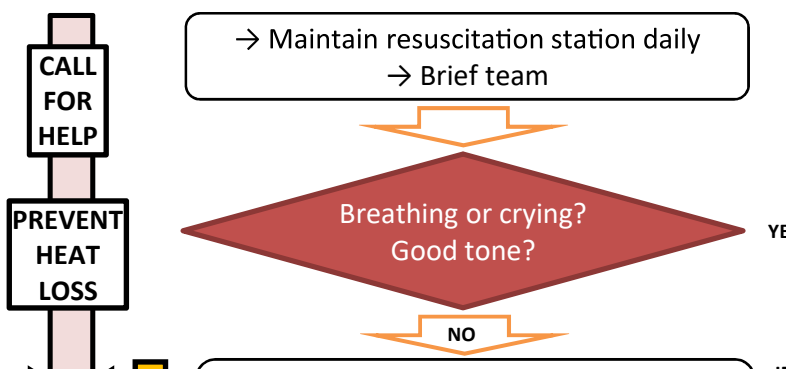
$\rightarrow$ Stimulate

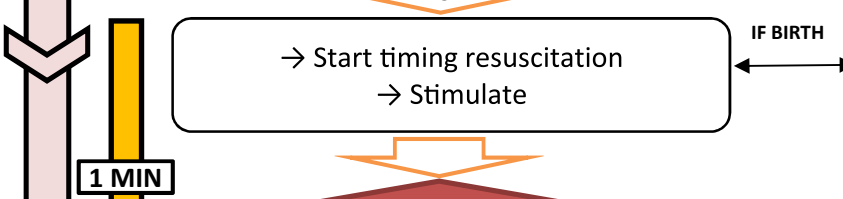

Breathing or crying? YES

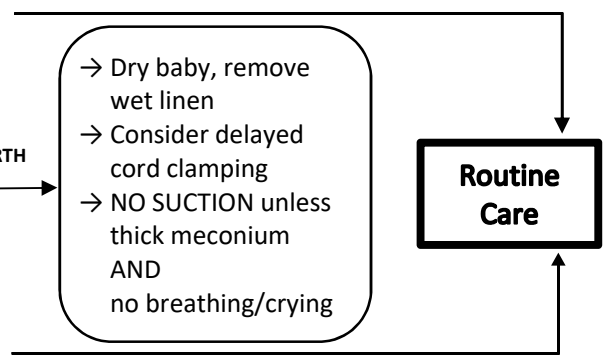

NO

Effective Ventilation for 30 seconds with room air
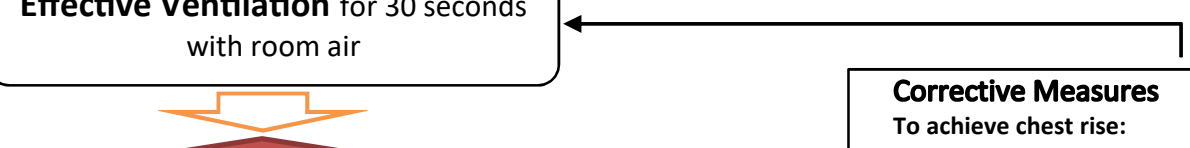

Chest moving?

No

To achieve chest rise:

1. Reposition mask and

reposition head

THEN:

2. Two person ventilation,

if staff allows

3. Increase pressure

$\rightarrow$ Check HR

$\rightarrow$ Consider adding $\mathrm{O}_{2}$ using pulse oximeter* (if available)

4. Consider oral airway
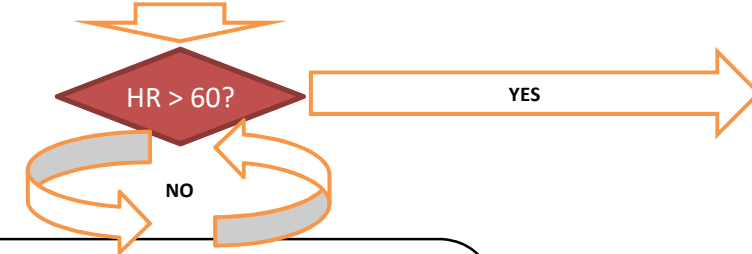

$\rightarrow$ Recheck HR every 1 minute

$\rightarrow$ Compressions + Ventilations (3:1) for $3 \mathrm{~min} /$ cycle

1st cycle: Prepare adrenaline IV $¥$ AND venous access (IV/IO/UVC) blood/volume + loss or pneumothorax

$>$ 3rd cycle: Consider stopping resuscitation $* *$ and provide comfort care

\begin{tabular}{|c|c|c|c|c|c|}
\hline \multicolumn{2}{|c|}{ Medication } & \multicolumn{4}{|c|}{ Weight } \\
\cline { 2 - 6 } & $\mathbf{1 ~ k g}$ & $\mathbf{2 ~ k g}$ & $\mathbf{3 ~ k g}$ & $\mathbf{4 ~ k g}$ \\
\hline $\begin{array}{c}\text { ₹ Adrenaline } \\
\mathbf{( 0 . 1} \mathbf{~ m g / m l}= \\
\mathbf{1 : 1 0 , 0 0 0 )}\end{array}$ & $\begin{array}{c}\text { Initial Dose } \\
(0.1 \mathrm{mg} / \mathrm{kg})\end{array}$ & $0.1 \mathrm{ml}$ & $0.2 \mathrm{ml}$ & $0.3 \mathrm{ml}$ & $0.4 \mathrm{ml}$ \\
\cline { 2 - 6 } & $\begin{array}{c}\text { Repeated } \\
\text { Dose }\end{array}$ & $0.1-0.3 \mathrm{ml}$ & $0.2-0.6 \mathrm{ml}$ & $0.3-0.9 \mathrm{ml}$ & $0.4-1.2 \mathrm{ml}$ \\
\hline $\begin{array}{c}\text { + Volume } \\
\text { Expansion }\end{array}$ & $\begin{array}{c}\text { NaCl } 0.9 \% \\
\text { (up to } 3 \\
\text { boluses) }\end{array}$ & $10 \mathrm{ml}$ & $20 \mathrm{ml}$ & $30 \mathrm{ml}$ & $40 \mathrm{ml}$ \\
\hline
\end{tabular}

* $\frac{\text { Minimum } \mathbf{O}_{2}}{\text { Saturation }}$
(per minute of life)
3 min: $70 \%$
$\quad 4 \min : 75 \%$
$5 \min : 80 \%$
$10 \min : 85 \%$

** Consider stopping resuscitation:

At 10 minutes: if no perceptible HR At 20 minutes: if no recovery of spontaneous ventilation, even if adequate $\mathrm{HR}$

Fig. 4. Progression of the NR algorithm throughout the consensus process: draft 12 and final version of the mid-level NR algorithm.

Designing a Mid-Level Neonatal Resuscitation Algorithm for MSF
Neonatology 2018;114:112-123

DOI: $10.1159 / 000486705$ 


\section{Acknowledgements}

The authors thank the MSF field workers, country-level coordinators, and headquarter team members who arranged for and participated in evaluation of the algorithm in clinical settings, in particular MSF-OCP projects in Katiola, Ivory Coast; Peshawar, Pakistan, and Aweil, South Sudan. Individual thanks are extended to fellow pediatricians for their feedback: Dr. Marco Olla, Dr. Johanna Thomson, and Dr. Belen Caminoa. The MSF International Pediatric Working Group is also thanked for its participation in algorithm finalization.

\section{Disclosure Statement}

None of the authors have a perceived conflict of interest or financial interests to disclose.

M.Bl. is a member of the Swedish Neonatal Society Working Group for Neonatal CPR. M.T. is a member of the Australian Resuscitation Council. M.T., D.T., and C.C.R. are ILCOR group members and D.T. and C.C.R. are ERC guideline authors. O.D.S. was part of the Guidelines Development Group for the 2012 WHO Guidelines on Basic Newborn Resuscitation.

The manuscript and algorithm have been endorsed by the European Society for Paediatric Research (ESPR) and the European Society for Neonatology (ESN, incorporated in ESPR since Oct. 2017). Authors M.Br. (ESN President until October 2017) and C.C.R. (ESPR President and former Officer of Education, ESN) are ESPR council members, and M.Bl. (former ESN President), M.T. (former Officer of Education, ESN) and O.D.S. (council member 1987-1990 and Secretary of the ESPR Working Group on Neonatology 1990-1993) are all active members of ESPR.

\section{Author Contributions}

L.U. conceived the concept of an independent MSF resuscitation algorithm. M.Bl. helped assemble the group of non-MSF neonatologists. M.Bl., M.Br., C.C.R., O.D.S., D.T., M.T., and L.U. devised the MSF resuscitation algorithm. A.B., C.C.R., M.T., and L.U. devised the draft manuscript. A.B., M.Bl., M.Br., C.C.R., O.D.S., D.T., M.T., and L.U. contributed to the completion of the manuscript.

\section{References}

1 Marshall S: The use of cognitive aids during emergencies in anesthesia: a review of the literature. Anesth Analg 2013;117:1162-1171.

2 McLanders ML, Marshall SD, Sanderson PM, Liley HG: The cognitive aids in medicine assessment tool (CMAT) applied to five neonatal resuscitation algorithms. J Perinatol 2017; 37:387-393.

3 Singhal N, Lockyer J, Fidler H, Keenan W, Little G, Bucher S, Qadir M, Niermeyer S: Helping Babies Breathe: global neonatal resuscitation program development and formative educational evaluation. Resuscitation 2012;83:90-86

4 Perlman JM, Wyllie J, Kattwinkel J, Wyckoff MH, Aziz K, Guinsburg R, Kim H, Liley HG, Mildenhall L, Simon WM, Szyld E, Tamura M, Velaphi S. Part 7: neonatal resuscitation: 2015 international consensus on cardiopulmonary resuscitation and emergency cardiovascular care science with treatment recommendations. Circulation 2015;132(suppl 1):S204-S241.

5 Roehr CC, Hansmann G, Hoehn T, Bührer C: The 2010 Guidelines on Neonatal Resuscitation (AHA, ERC, ILCOR): similarities and differences - what progress has been made since 2005? Klin Padiatr 2011;223:299-307.

6 Mileder LP, Urlesberger B, Szyld EG, Roehr CC, Schmölzer GM: Simulation-based neonatal and infant resuscitation teaching: a systematic review of randomized controlled trials. Klin Padiatr 2014;226:259-267.
7 Dempsey E, Pammi M, Ryan A, Barrington K: Standardised formal resuscitation training programmes for reducing mortality and morbidity in newborn infants. Cochrane Database Syst Rev 2015;9:CD009106.

8 Wyllie J, Bruinenberg J, Roehr CC, Rüdiger M, Trevisanuto D, Urlesberger B: European Resuscitation Council Guidelines for Resuscitation 2015: Section 7. Resuscitation and support of transition of babies at birth. Resuscitation 2015;95:249-263.

9 American Academy of Pediatrics: Neonatal Resuscitation Program. https://www.aap.org/ en-us/continuing-medical-education/lifesupport/NRP/Pages/NRP.aspx (accessed June 17, 2017).

10 Wyckoff MH, Aziz K, Escobedo MB, Kapadia VS, Kattwinkel J, Perlman JM, Simon WM, Weiner GM, ZaichKin JG: Part 13: neonatal resuscitation: 2015 AHA guidelines update for cardiopulmonary resuscitation and emergency cardiovascular care. Circulation 2015; 132(suppl 2):S543-S560.

11 American Academy of Pediatrics: Helping Babies Breathe, ed 2. Itasca, AAP, 2016. Available at https://www.aap.org/en-us/advocacyand-policy/aap-health-initiatives/helpingbabies-survive/Pages/Helping-BabiesBreathe-Edition.aspx (accessed April 28, 2017).
12 Wall SN, Lee ACC, Carlo W, Goldenberg R, Niermeyer S, Darmstadt GL, Keenan W, Bhutta ZA, Perlman J, Lawn JE: Reducing intrapartum-related neonatal deaths in lowand middle-income countries - what works? Semin Perinatol 2010;34:395-407.

13 Médecins Sans Frontières (MSF): International Activity Report 2016. Geneva, MSF, 2017.

14 Kak LP, Johnson J, McPherson R, Keenan W, Schoen E (eds): Helping Babies Breathe: Lessons Learned Guiding the Way Forward. HBB Global Development Alliance, 2015. http:// cdn.laerdal.com/downloads-test/f3790/ HBB_report_2010-2015_FINAL.pdf (accessed May 15, 2017).

15 World Health Organization: Guidelines on Basic Newborn Resuscitation. Geneva, WHO, 2012.

16 Newton O, English M: Newborn resuscitation: defining best practice for low income settings. Trans R Soc Trop Med Hyg 2006; 100:899-908

17 Saugstad OD, Robertson NJ, Vento M: A critical review of the 2015 International Liaison Committee on Resuscitation treatment recommendations for resuscitating the newly born infant. Acta Paediatr 2016;105:442-444.

18 Klingenberg C, O'Donnell CP: Insuflation breaths - a transatlantic divide in guidelines for neonatal resuscitation. Resuscitation 2016;101:e19. 
19 Larcher V, Craig F, Bhogal K, Wilkinson D, Brierley J: Making decisions to limit treatment in life-limiting and life-threatening conditions in children: a framework for practice. Arch Dis Child. 2015;100(suppl 2):s1-s26.

20 Andersson O, Hellstrom-Westas L, Andersson D, Domellof M: Effect of delayed versus early umbilical cord clamping on neonatal outcomes and iron status at 4 months: a randomised controlled trial. BMJ 2011; 343:d7157.

21 Ashish KC, Rana N, Malqvist M, Ranneberg LJ, Subedi K, Andersson O: Effects of delayed umbilical cord clamping vs early clamping on anemia in infants at 8 and 12 months. JAMA Pediatr 2017;171:264-270.

22 Berkelhamer SK, Kamath-Rayne BD, Niermeyer S: Neonatal resuscitation in low-resource settings. Clin Perinatol 2016;43:573591.

23 Chaparro CM, Neufeld LM, Alavez GT, Cedillo ELR, Dewey KG: Effect of timing of umbilical cord clamping on iron status in Mexican infants: a randomised control trial. Lancet 2006;367:1997-2004.
24 Manley BJ, Owen LS, Hooper SB, Jacobs SE, Cheong JLY, Doyle LW, Davis PG: Towards evidence-based resuscitation of the newborn infant. Lancet 2017;389:1639-1648.

25 Van Rheenen P, De Moor L, Eschback S, De Grooth H, Brabin B: Delayed cord clamping and haemoglocin levels in infancy: a randomised controlled trial in term babies. Trop Med Int Health 2007;12:603-616.

26 Saugstad OD, Ramji S, Soll RF, Vento M: Resuscitation of newborn infants with $21 \%$ or $100 \%$ oxygen: an updated systematic review and meta-analysis. Neonatology 2008;94: 176-182.

27 Haines M, Wright IM, Bajuk B, Abdel-Latif ME, Hilder L, Challis D, Guaran R: Population-based study shows that resuscitating apparently stillborn extremely preterm babies is associated with poor outcomes. Acta Paediatrica 2016;105:1305-1311.

28 Harrington DJ, Redman CW, Moulden M, Greenwood CE: The long-term outcome in surviving infants with Apgar zero at $10 \mathrm{~min}$ utes: a systematic review of the literature and hospital-based cohort. Am J Obstet Gynecol 2007;196:463.463.e5.
29 Jain L, Ferre C, Vidyasagar D, Nath S, Sheftel D: Cardiopulmonary resuscitation of apparently stillborn infants: survival and long-term outcome. J Pediatr 1991;118:778-782.

30 McGrath JS, Roehr CC, Wilkinson DJC: When should resuscitation at birth cease? Early Hum Dev 2016;102:31-36.

31 Shah P, Anvekar A, McMichael J, Rao S: Outcomes of infants with Apgar score of zero at 10 min: the West Australian experience. Arch Dis Child Fetal Neonatal Ed 2015;100:F492F494.

32 Limitations of resuscitation. Lancet 1972;1: 1169-1170.

33 Travers CO, Carlo WA: How to save 1 million lives in a year in low- and middle-income countries. Neonatology 2017;111:431-436.

34 Fuerch JH, Yamada NK, Coelho PR, Lee HC, Halamek LP: Impact of a novel decision support tool on adherence to neonatal resuscitation program algorithm. Resuscitation 2015; $88: 52-56$ 